\title{
Within- and across-channel factors in the multiband comodulation masking release paradigm
}

\author{
John H. Grose, ${ }^{\text {a) }}$ Emily Buss, and Joseph W. Hall III \\ Department of Otolaryngology/Head and Neck Surgery, University of North Carolina at Chapel Hill, \\ Chapel Hill, North Carolina 27599-7070
}

(Received 12 November 2007; revised 20 October 2008; accepted 21 October 2008)

\begin{abstract}
Maskers made up of comodulated narrow bands of noise can result in a signal detection advantage due to both within- and across-channel processes. The purpose of this study was to determine whether contributions from these processes could be differentiated on the basis of two stimulus manipulations: (1) onset/offset asynchrony across bands and (2) introduction of a random temporal fringe surrounding the comodulated bands. The hypothesis was that only masking release due to across-channel processing would be disrupted by these manipulations. Five-band comodulated maskers were constructed, and the availability of within- and across-channel cues was varied by adjusting the frequency spacing of the bands; both logarithmic and linear spacings were tested. The signal was a $1 \mathrm{kHz}$ pure tone. Onset/offset asynchrony had different effects depending on the characteristics of the asynchrony. The results were consistent with an interpretation that across-channel, but not within-channel, masking release was disrupted when the flanking bands were presented continuously and the on-signal band was gated. However, the results suggested that both the across-channel and the within-channel masking release were disrupted in conditions where the on-signal band was continuous and the flanking bands were gated on, as well as in conditions where a random temporal fringe was present.
\end{abstract}

(C) 2009 Acoustical Society of America. [DOI: 10.1121/1.3023067]

PACS number(s): 43.66.Dc, 43.66.Mk [BCM]

Pages: 282-293

\section{INTRODUCTION}

The detection of a tonal signal masked by a narrow band of noise centered at the signal frequency can be facilitated by the presence of additional narrow bands of noise that share the same fluctuation pattern as the on-signal masking band. This detection advantage is termed comodulation masking release (CMR), and the measurement technique of employing a complex of comodulated narrow bands of noise will be referred to here as the multiband CMR paradigm. The phenomenon of CMR has been used as an exemplar of acrosschannel processing in the auditory system, where such processing refers to the synthesis of information transduced in independent (peripheral) frequency channels. In the case of multiband CMR, across-channel processing refers to the use of signal detection cues that derive from the comparison of concurrent stimulus patterns in spectrally discrete regions (for review, see Verhey et al., 2003; Grose et al., 2005a). However, as will be expanded on below, it is evident that within-channel cues can provide some, if not most, of the detection information under some conditions. Here, the detection cues are derived from information contained within the output of a single auditory filter. It is therefore important for the understanding of CMR to be able to distinguish the contributions of within- and across-channel cues to signal detection. The purpose of this study is to assess two manipulations that have been purported to assist in this differentiation using the multiband CMR paradigm.

${ }^{\text {a)} E l e c t r o n i c ~ m a i l: ~ j h g @ m e d . u n c . e d u ~}$
The initial demonstration of CMR contributed to a growing interest in across-channel mechanisms in signal detection (Hall et al., 1984), but it was noted early on that within-channel cues could contribute strongly to the detection advantage in the multiband CMR paradigm under some conditions (Schooneveldt and Moore, 1987). In their study, Schooneveldt and Moore (1987) demonstrated that the magnitude of masking release was sensitive to the frequency proximity of the (single) comodulated flanking band (CFB), with the largest masking releases occurring for close spacing of the two bands. It was argued that these local threshold minima reflected sensitivity to signal-induced disruptions in temporal beating patterns between the two comodulated bands; i.e., a within-channel cue. One proposed manifestation of this cue, among several considered by Schooneveldt and Moore (1987), was a change in the pattern of phase locking brought about by the signal dominating the neural synchrony during the minima of the beating masker. Singlechannel cues for CMR based on temporal envelope interactions have also been proposed by Berg (1996). ${ }^{1}$ The extent to which masking release measured with the multiband CMR paradigm represents an across-channel process, therefore, depends on the spectral relations (and levels) of the comodulated noise bands.

In many studies, the assumption of across-channel processing in CMR is implicit in the selection of the frequency spacings of the multiple narrow bands of noise making up the comodulated masker: placement of the individual bands in putatively independent frequency channels is usually assumed to facilitate primarily across-channel processing. However, the assumption of channel independence is often 
not systematically tested. One approach to differentiating within- and across-channel factors is based on the supposition that facets of auditory grouping and across-channel processing are intrinsically linked, whereas this is not the case for within-channel processing. That is, the perceptual organization of a complex sound involves in part the extraction of common features from across the spectrum (e.g., common amplitude modulation), and this extraction constitutes a form of across-channel processing. The notion of an interdependency between across-channel processing and auditory grouping implies that factors resulting in a disruption of auditory grouping have their effect by disrupting acrosschannel processing but should have no effect on withinchannel processing. This rationale has been applied in several contexts where the role of across-channel processing has been explored by manipulating strength of auditory grouping through the use of temporal asynchrony. For example, the question of whether modulation detection interference (MDI) ${ }^{2}$ results from a perceptual fusion of the modulating probe and the distal interferer has been tested by introducing gating asynchronies between probe and interferer to weaken their perceptual fusion (Hall and Grose, 1991; Moore and Shailer, 1992; Oxenham and Dau, 2001). Similarly, gating asynchronies have been used to assess the contribution of within- and across-channel processing to comodulation difference detection $(\mathrm{CDD})^{3}$ (Moore and Borrill, 2002; Hall et al., 2006).

For multiband CMR, Grose and Hall (1993) showed that the magnitude of masking release was dependent on the gating synchrony between the on-signal masking band and the accompanying CFBs: onset asynchrony across bands elevated threshold for detecting the signal despite the fact that the masker configuration during the listening interval was unaffected by this manipulation. They interpreted this effect as being due to a disruption of the perceptual fusion between the on-signal band (OSB) and the CFBs, and concluded that auditory grouping processes are integral to the acrosschannel mechanisms underlying CMR. Dau et al. (2005) extended this result by applying the gating asynchrony manipulation to complexes of comodulated narrow bands of noise that were specifically selected to be either proximal in frequency (increasing availability of within-channel cues) or well separated in frequency (decreasing availability of within-channel cues). In some conditions the on-signal and flanking masker bands were synchronously gated, and in other conditions the flanking bands were gated on $100 \mathrm{~ms}$ prior to OSB onset. Results demonstrated that this gating asynchrony reduced masking release for the widely spaced complexes but not for the narrowly spaced complexes. This finding was also interpreted in the context of auditory grouping, viz., across-channel mechanisms that contribute to CMR are integral to auditory grouping processes in perceptual organization.

Another approach that has been used to shed light on the nature of multiband CMR involves manipulation of masker modulation coherence before and after the comodulated listening interval (Mendoza et al., 1998; Grose et al., 2005b). In this approach, signal detection is measured in a complex of synchronously gated comodulated bands of noise as a function of the characteristics of the noise presented during the interstimulus and intertrial intervals. When this temporal fringe consists of noise bands that are in all respects the same as the comodulated bands, with the exception that their envelopes are independent of each other (random), masking release declines relative to that measured in the absence of the fringe (or relative to that measured with a comodulated fringe). Note that this effect relies on a seamless perceptual continuity between the fringe and the comodulated interval; i.e., the transition from fringe to comodulated interval is perceptually transparent (Mendoza et al., 1998). One way to view the effect of the random temporal surround is that, during the periods outside of the observation intervals, the uncorrelated envelope fluctuations across the random masker might closely resemble across-channel difference cues used to detect the presence of a signal. Because these spurious signal-like cues occur so frequently in the fringe condition, the perceptual weight given to such cues could be reduced, an underweighting that persists into the comodulated observation intervals. Grose et al. (2005b) proposed that this sensitivity to the random temporal surround was a trait of across-channel CMR and could, in consequence, be used as a criterion test. In that study, the bands of noise were well separated in frequency, and so it was implicitly assumed that the masking release was due to across-channel processing. However, before it can be concluded that the random temporal fringe manipulation constitutes a criterion test for acrosschannel CMR, it should be demonstrated that this manipulation does not affect within-channel processing. That is, if the temporal fringe manipulation is a test for across-channel CMR, then it should have no effect on the masking release measured using closely spaced noise bands, which presumably results mainly from within-channel processing. This reasoning is analogous to the logic used by Dau et al. (2005) concerning the role of gating asynchronies and auditory grouping in CMR.

The purpose of this study was to further test manipulations that differentially affect within- versus across-channel masking release. Two specific issues were addressed. First, the effect of a random temporal fringe on within-channel processing was assessed to determine the validity of the proposition that this manipulation constitutes a test for across-channel CMR. The second specific issue is the effect of gating asynchronies on within-channel processing. As noted above, Dau et al. (2005) provided an important extension to the Grose and Hall (1993) study by testing stimulus configurations designed to accentuate within-channel interactions. However, they tested only conditions where the CFBs were gated on before, and gated off after, the OSB. Whereas they found a reduction-but not an elimination-of the masking release in the across-channel conditions, as did Grose and Hall (1993), they did not test the inverse conditions where the OSB was gated on before, and gated off after, the flanking bands. ${ }^{4}$ This configuration was particularly informative in the Grose and Hall (1993) study since it showed a complete elimination of the masking release for asynchronies greater than about $50 \mathrm{~ms}$. A goal of this experiment, therefore, was to include this configuration in a test of 
gating asynchrony effects across a range of frequency spacings that would encompass both within- and across-channel processes.

In summary, this study tests the hypothesis that multiband CMR sometimes occurs as a result of across-channel processes, and that these processes are intrinsically linked with auditory grouping. The hypothesis was tested by assessing the effects of two specific stimulus manipulations on masking release: (1) onset/offset asynchronies across the bands making up the comodulated complex and (2) embedding the gated comodulated complex into a random temporal fringe. Both manipulations were tested using comodulated complexes where the noise bands were variably spaced in frequency to preferentially provide either within- or acrosschannel cues. Two experiments were undertaken. The first adopted and extended the approach of Dau et al. (2005) which employed predominantly logarithmic spacing of masker bands. The second experiment used linear spacing of masking bands. The investigation of linear spacing was of interest because within-channel cues in such configurations are likely to be more salient than in logarithmic spacing, an issue developed further in the preamble to experiment 2 , below.

\section{EXPERIMENT 1. MULTIBAND CMR FOR LOGARITHMIC SPACING}

\section{A. Method}

\section{Observers}

Observers were five highly practiced normal-hearing adults (two females). All had pure tone thresholds $\leqslant 20 \mathrm{~dB}$ $\mathrm{HL}$ at octave frequencies from $250-8000 \mathrm{~Hz}$, and none reported a history of significant ear disease. Ages ranged from 24 to 54 years (mean of 38 years).

\section{Stimuli}

The signal in all conditions was a $1000 \mathrm{~Hz}$ pure tone, $280 \mathrm{~ms}$ in duration including $40 \mathrm{~ms}$ raised-cosine onset/ offset ramps. In all conditions, the signal was masked by a $20 \mathrm{~Hz}$ wide band of Gaussian noise centered at $1000 \mathrm{~Hz}$. This OSB was either presented alone or was accompanied by four additional $20 \mathrm{~Hz}$ wide bands of noise, two centered above and two centered below the OSB frequency. The bands were logarithmically spaced. The center frequencies were 944, 972, 1000, 1029, and $1059 \mathrm{~Hz}$ (1/24th-octave); 794, 891, 1000, 1122, and $1260 \mathrm{~Hz}$ (1/6th-octave); 630, 794, 1000, 1260, and $1587 \mathrm{~Hz}$ (1/3rd-octave); and 250, 500, 1000,2000 , and $4000 \mathrm{~Hz}$ (1-octave). These frequency spacings were selected to span the range from wholly subcritical (falling within the bandwidth of a single auditory filter centered at $1000 \mathrm{~Hz}$ ) to maximal frequency independence (each band falling within the passband of a separate auditory filter). The $1 / 6$ th-octave spacing and the 1-octave spacing are the same as those used by Dau et al. (2005) to exemplify within- and across-channel configurations, respectively. Each masker band was presented at $60 \mathrm{~dB}$ sound pressure level (SPL).

A total of 30 masking conditions were constructed: 6 reference conditions and 24 conditions employing comodu-
TABLE I. Summary of masker conditions. Core/Surround denotes the characteristics of the masker during the observation interval (Core) and during the period outside of the observation intervals (Surround). These characteristics were either absent masker $(\varnothing)$, on-signal band alone (OSB), comodulated flanking bands alone (CFB), five-band comodulated complex (COM), five-band codeviant complex (COD), or five-band random complex (RAN). Where four groups of conditions are indicated in a row, this indicates that the same Core/Surround was applied for each of the four frequency spacings $(1 / 24$ th-, $1 / 6$ th- $1 / 3$ rd-, and 1-octave). The table also indicates the condition classification.

\begin{tabular}{lcl}
\hline \hline Cond & Core/Surround & \multicolumn{1}{c}{ Classification } \\
\hline 1 & OSB/ $\varnothing$ & Reference (gated OSB) \\
2 & OSB/OSB & Reference (continuous OSB) \\
$3-6($ Exp 1) & RAN/RAN & Reference (continuous random bands) \\
$3-6$ (Exp 2) & RAN/ $\varnothing$ & Reference (gated random bands) \\
$7-10$ & COM/ $\varnothing$ & Baseline (gated comodulated bands) \\
$11-14$ & COM/OSB & Asynchrony (continuous OSB) \\
$15-18$ & COM/CFB & Asynchrony (continuous CFBs) \\
$19-22$ & COM/RAN & Fringe (random) \\
$23-26$ & COM/COD & Fringe (codeviant) \\
$27-30$ & COM/COM & Fringe (comodulated) \\
\hline \hline
\end{tabular}

lated five-band maskers during the observation intervals. These conditions are summarized in Table I and shown schematically in Fig. 1. The nomenclature for all conditions used in this study pairs the characteristics of the masker during the observation intervals (the Core) with the characteristics of the masker during the periods outside of the observation intervals (the Surround). Thus, each condition is designated by a [Core/Surround] label. Two of the reference conditions consisted of the OSB presented alone. In one (Cond 1), it was gated on in the listening intervals for $280 \mathrm{~ms}$, including $40 \mathrm{~ms}$ onset/offset raised-cosine ramps (Core=OSB; Surround $=$ absent masker, or $\oslash$; i.e., $[\mathrm{OSB} / \oslash])$. The signal, when it occurred, was therefore gated concurrently with the masker. The second reference condition (Cond 2) consisted of the OSB presented continuously [OSB/OSB]. The remaining four reference conditions (Conds 3-6) consisted of fiveband maskers presented continuously, where each band was random with respect to the others [RAN/RAN]. There was one $[\mathrm{RAN} / \mathrm{RAN}]$ condition for each of the four frequency spacings (1/24th-, 1/6th-, 1/3rd-, and 1-octave).

The remaining 24 conditions fell into three sets. The first set consisted of the baseline comodulated conditions (Conds 7-10). Here, the five-band comodulated masker complexes were gated on for $280 \mathrm{~ms}$, including $40 \mathrm{~ms}$ onset/offset raised-cosine ramps, during the listening intervals $[\mathrm{COM} / \oslash]$. There were four conditions in this set, one for each of the four frequency spacings. The second set of eight conditions addressed the factor of onset/offset synchrony across bands. In four of the conditions (Conds 11-14), one for each of the frequency spacings, the OSB was presented continuously and the CFBs were gated on during the $280 \mathrm{~ms}$ listening intervals $[\mathrm{COM} / \mathrm{OSB}]$. In the remaining four of the eight conditions (Conds 15-18), the reverse occurred: for each of the four frequency spacings, the CFBs were presented continuously and the OSB was gated on during the $280 \mathrm{~ms}$ listening intervals $[\mathrm{COM} / \mathrm{CFB}]$. The third set of conditions addressed the factor of a temporal fringe and contained 12 


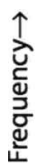

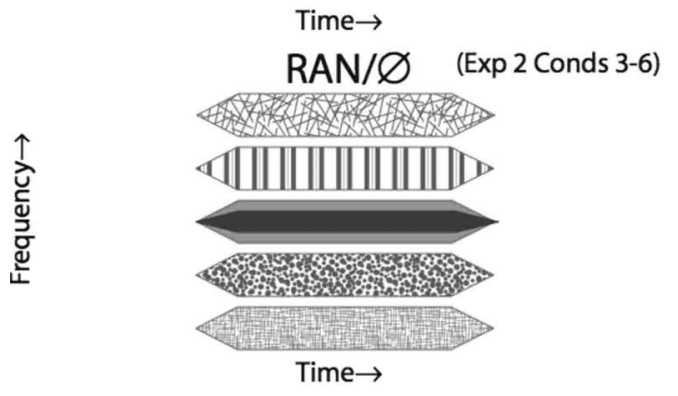

$\mathrm{COM} / \varnothing$

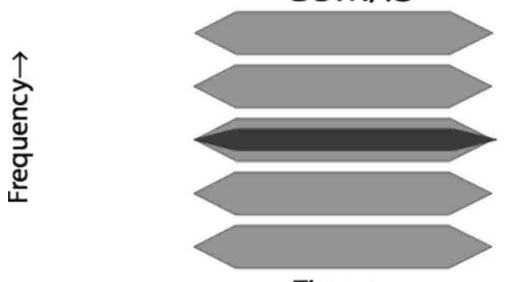

Time $\rightarrow$

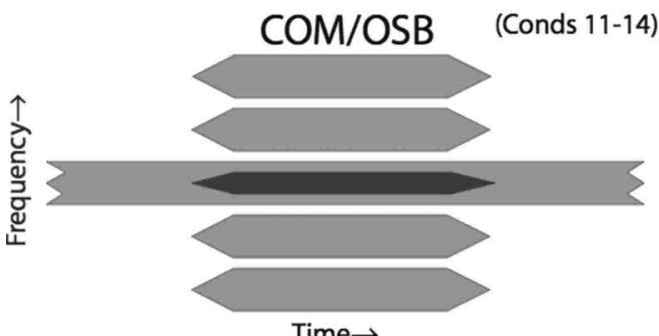

Time $\rightarrow$

COM/RAN

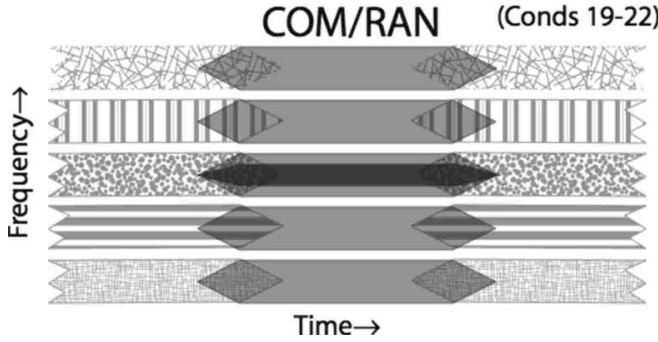

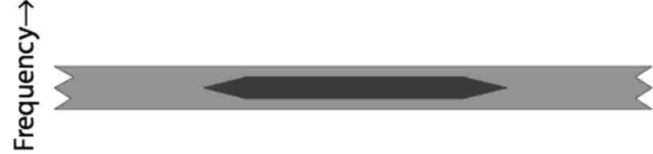

Time $\rightarrow$
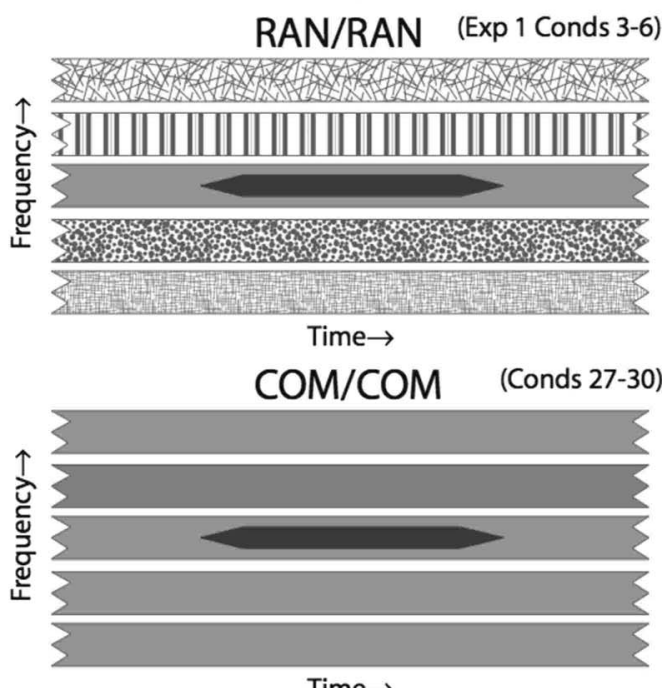

Time $\rightarrow$

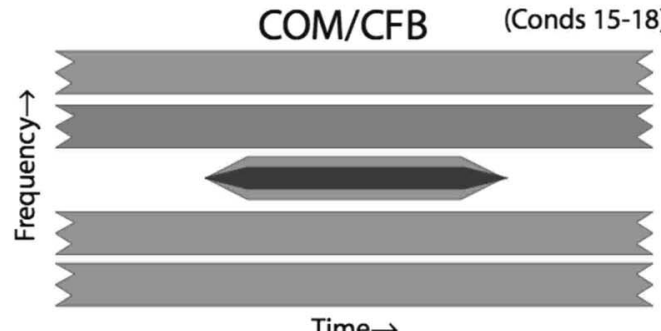

Time $\rightarrow$

COM/COD (Conds 23-26)

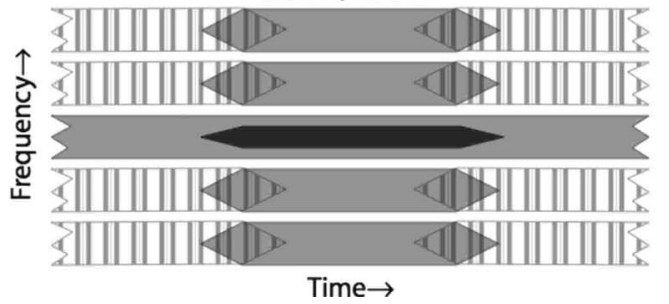

FIG. 1. Schematic representations of the stimulus conditions. Each panel shows a Core/Surround configuration for the masker (see also Table I). The signal is denoted by a black trapezoid; identical fill patterns across masker bands indicate comodulation.

conditions. In 4 of the 12 conditions (Conds 19-22), one for each of the frequency spacings, the five-band comodulated masker was gated on during the listening intervals, but these core comodulated intervals were temporally surrounded by a continuous presentation of five random noise bands that were in all respects the same as the comodulated bands except that each band had an independent fluctuation pattern from all others $[\mathrm{COM} / \mathrm{RAN}]$. The transition from random fringe to comodulated listening interval was perceptually seamless and was implemented by overlapping $40 \mathrm{~ms}$ gates; i.e., the fringe was gated off as the comodulated core was gated on to begin the listening interval, and the random fringe was gated on as the comodulated core was gated off at the end of the interval. In the next four conditions (Conds 23-26), the tem- poral fringe consisted of codeviant bands [COM/COD]. Here, the four flanking bands were comodulated with respect to each other, but independently of the OSB. The rationale for this configuration was that, under conditions where across-frequency processing facilitated auditory grouping behavior, the flanking bands might form a separate auditory stream from the OSB, thus perceptually isolating the OSB. Thus, performance in the $[\mathrm{COM} / \mathrm{COD}]$ configuration might show a dependency on frequency spacing that reflected within- versus across-frequency processing. In the final four conditions (Conds 27-30), the temporal fringe consisted of five comodulated bands [COM/COM]. These conditions are functionally equivalent to the presentation of continuous comodulated noise bands because there was no perceptual dis- 
tinction between the comodulated temporal fringe and the comodulated core. This configuration allowed for a comparison between gated and continuous comodulated bands as a function of frequency spacing. A gated/continuous difference is characteristic of CMR (Hatch et al., 1995).

Maskers were generated in the frequency domain based on $2^{17}$ points. When played out at a sampling rate of $12207 \mathrm{~Hz}$, this resulted in a waveform segment $10.7 \mathrm{~s}$ in duration that repeated seamlessly when played continuously. The coherence or independence of bands across frequency was controlled via the assignment of values to the real and imaginary components in the spectral domain. To generate a single noise band, those points in the complex spectrum corresponding to components within the desired passband were assigned values drawn from a Gaussian distribution; all other points were set to zero. To generate comodulated bands, the same set of random draws was used to define the real and imaginary components of each of the five bands. To generate bands with independent envelope fluctuations across bands, each of the five bands received random values for the real and imaginary components (i.e., independent draws from a Gaussian distribution for each band). To generate bands for the codeviant fringe conditions, one set of random draws was used to define the OSB and a second set of independent draws was used to define the remaining four flanking maskers. Two sets of maskers were generated prior to each threshold estimation track, one set for presentation during the listening intervals and one set for presentation during the interstimulus and intertrial intervals. In conditions where no fringe stimulus was required, the array defining the fringe masker was filled with zeros. Stimulus output and gating were controlled by a digital signal processing platform [Tucker-Davis Technologies (TDT) RP2]. The stimulus was routed through a headphone buffer (TDT HB7) and presented to the left phone of a Sennheiser 265 Linear headset.

\section{Procedure}

Stimuli were presented in a three-alternative forcedchoice paradigm, with a $280 \mathrm{~ms}$ observation interval and a $500 \mathrm{~ms}$ interstimulus interval. Each observation interval was visually marked by a light on a response box. Observers entered their response after each trial by means of a button on the response box and correct interval feedback was provided by means of lights. Signal thresholds were estimated using a three-down one-up track that converged on the $79 \%$ correct point on the psychometric function. Signal level was adjusted in steps of $4 \mathrm{~dB}$, reduced to $2 \mathrm{~dB}$ after the second track reversal, and a track continued until eight reversals were obtained. The threshold estimate for a track was the mean signal level at the last six reversals. Three threshold estimates were collected for each condition. If the range of these estimates exceeded $3 \mathrm{~dB}$, a fourth estimate was collected. The final threshold value for a condition was taken as the arithmetic mean of the three (or four) estimates.

\section{B. Results and discussion}

The results of the reference and baseline conditions are shown in Fig. 2. All five observers exhibited similar patterns

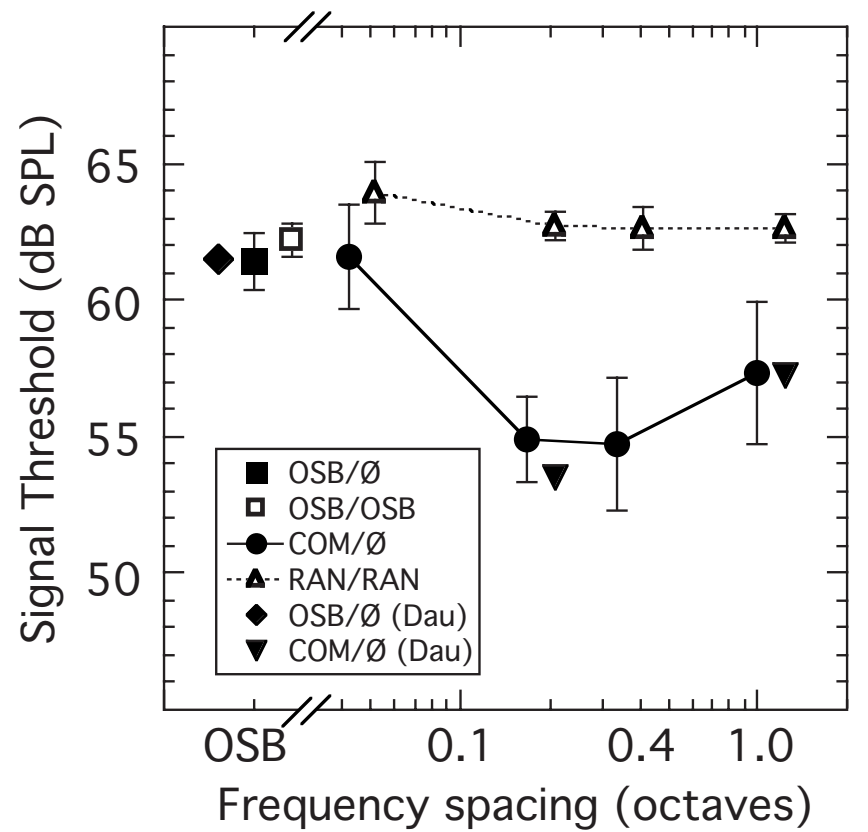

FIG. 2. Mean results for reference and baseline conditions from experiment 1. OSB alone thresholds are shown as filled square for gated presentation (i.e., $[\mathrm{OSB} / \oslash]$ ) and unfilled square for continuous presentation (i.e., [OSB/ $\mathrm{OSB}]$ ); for comparison, filled diamond is [OSB/ $\oslash$ ] from Dau et al. (2005). Thresholds in continuous random maskers [RAN/RAN] as a function of band spacing are shown by open triangles. Thresholds in gated comodulated maskers $[\mathrm{COM} / \oslash]$ are shown as circles; for comparison, filled inverted triangles are $[\mathrm{COM} / \oslash]$ from Dau et al. (2005). Error bars are \pm 1 standard deviation.

of performance, and so only group means and standard deviations are shown. The filled and unfilled squares indicate, respectively, thresholds in the OSB masker presented alone in gated $[\mathrm{OSB} / \oslash]$ and continuous $[\mathrm{OSB} / \mathrm{OSB}]$ modes. The unfilled triangles indicate thresholds in the continuous random bands [RAN/RAN]. Filled circles are thresholds for the gated comodulated bands $[\mathrm{COM} / \oslash]$. Also shown are corresponding data from Dau et al. (2005) for the gated OSB alone (diamond) and gated comodulated bands (inverted triangles). A preliminary consideration concerns the choice of reference condition for assessing masking release associated with the comodulated masker. For the OSB alone, thresholds were equivalent for the gated and continuous presentation modes $\left(t_{4}=1.589 ; p=0.19\right)$. Masking release referenced to the gated OSB was assessed by comparing thresholds for the $[\mathrm{OSB} / \oslash]$ condition and the four $[\mathrm{COM} / \oslash]$ conditions using a repeated measures analysis of variance (ANOVA). The analysis showed a significant overall effect of condition $\left(F_{4,16}=32.95 ; p<0.001\right)$. Simple contrasts indicated that the masking release was significant for the three wider flanking band spacings $\left(F_{1,4}\right.$ ranging from 19.32 to $109.59 ; p$ $<0.012)$; no masking release occurred for the narrowest spacing $\left(F_{1,4}=0.08 ; p=0.80\right)$. Masking release referenced to the continuous random bands was assessed by comparing thresholds for the [RAN/RAN] and [COM/ $\oslash$ conditions. The repeated measures ANOVA indicated a significant effect of masker type $\left(F_{1,4}=63.825 ; p<0.001\right)$, a significant effect of frequency spacing $\left(F_{1,4}=26.523 ; p<0.001\right)$, and a significant interaction between these two factors $\left(F_{3,12}=21.911 ; p\right.$ $<0.001)$. Analysis of simple effects showed that for each 


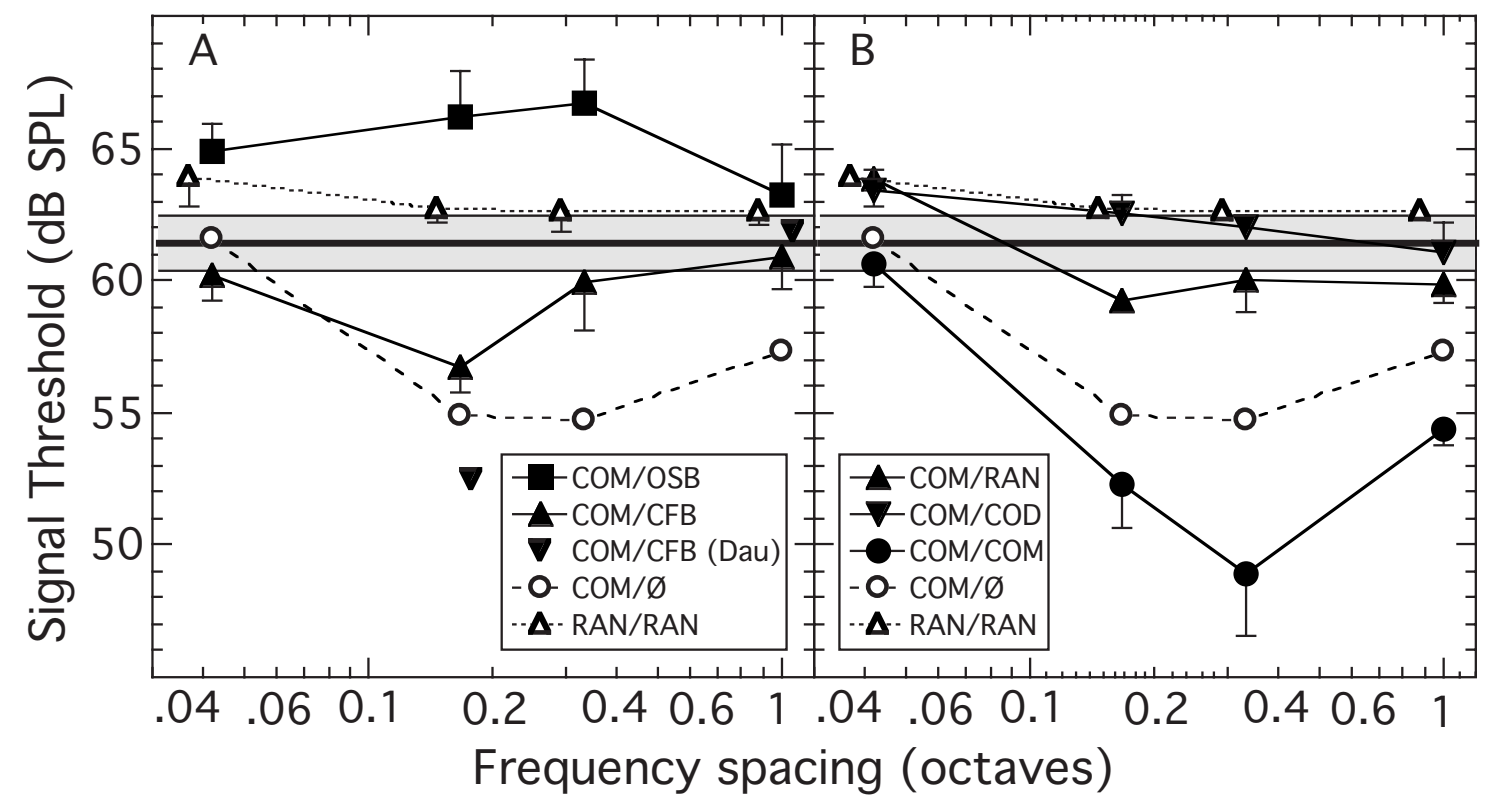

FIG. 3. Mean results for asynchrony conditions (Panel A) and temporal fringe conditions (Panel B) from experiment 1. Panel A: filled squares $=[\mathrm{COM} / \mathrm{OSB}]$; filled triangles $=[\mathrm{COM} / \mathrm{CFB}]$; filled inverted triangles $=[\mathrm{COM} / \mathrm{CFB}]$ from Dau et al. $(2005)$. Panel B: filled triangles $=[\mathrm{COM} / \mathrm{RAN}]$; filled inverted triangles $=[\mathrm{COM} / \mathrm{COD}]$; filled circles $=[\mathrm{COM} / \mathrm{COM}]$. In each panel, replotted from Fig. 2, are the $[\mathrm{OSB} / \oslash]$ reference (the thick horizontal line bounded by a \pm 1 standard deviation shaded region), the [RAN/RAN] reference (open triangles), and the [COM/OSB] baseline (open circles). Error bars are 1 standard deviation.

frequency spacing thresholds in the comodulated masker were significantly lower than in the random masker $(p$ $<0.02)$. For the three wider frequency spacings $(1 / 6$ th-, $1 / 3$ rd-, and 1-octave), therefore, a significant masking release was observed irrespective of whether the reference was the OSB alone or the five-band random masker. However, for the narrowest frequency spacing (1/24th-octave), a significant masking release was observed only when the five-band random masker was used as the reference. (The higher signal threshold in the five-band random masker spaced at $1 / 24$ th-octave relative to the single-band OSB masker presumably reflects increased energetic masking.) Because of the precedence of using the OSB as the reference for masking release in this paradigm (Grose and Hall, 1993; Dau et al., 2005), this reference will also serve as the primary anchor in this study. However, where the pattern of data differs depending on which reference is used, consideration of the five-band random masker will also be included.

One stimulus manipulation designed to reduce the strength of auditory grouping among the comodulated noise bands was that of introducing an onset/offset asynchrony across the bands. The asynchrony was introduced in two ways: (1) the OSB was presented continuously and the CFBs were gated on $[\mathrm{COM} / \mathrm{OSB}]$; and (2) the CFBs were presented continuously and the OSB was gated on [COM/CFB]. The results for this pair of conditions are shown in Fig. 3, Panel A (filled symbols). For comparison, reference thresholds and baseline thresholds for synchronously gated comodulated bands are replotted from Fig. 2: the $[\mathrm{OSB} / \oslash]$ reference threshold is shown as a thick horizontal line with the shaded region indicating \pm 1 standard deviation; the [RAN/ RAN] reference thresholds are shown as open triangles; the $[\mathrm{COM} / \oslash]$ baseline thresholds are shown as open circles. Relative to the baseline gated masking release conditions, signal thresholds appear generally elevated when the OSB is presented continuously and the flanking bands are gated ([COM/OSB], filled squares). This increased masking appears not only to negate any masking release but to increase thresholds above reference thresholds for most spacings. When the flanking bands were presented continuously and the OSB was gated ([COM/CFB], filled triangles), thresholds appear higher than for the baseline masking release conditions in some cases and approach the reference thresholds for the two widest masker spacings. For comparison, data from Dau et al. (2005) for [COM/CFB] are also shown (inverted triangles).

In order to test the significance of these data patterns, separate repeated measures ANOVAs were undertaken for each mode of onset asynchrony. For the $[\mathrm{OSB} / \oslash]$ reference and the asynchrony with the OSB presented continuously $[\mathrm{COM} / \mathrm{OSB}]$, there was a significant overall effect of condition $\left(F_{4,16}=13.94 ; p<0.001\right)$. Post hoc simple contrasts indicated that thresholds in the asynchronous conditions were elevated relative to that for the OSB alone for all frequency spacings except the widest 1-octave spacing $\left(F_{1,4}\right.$ $=20.27-36.08 ; p<0.01)$. A similar pattern was found using the [RAN/RAN] reference except that a threshold elevation was absent also for the narrowest $1 / 24$ th-octave spacing. The $[\mathrm{COM} / \mathrm{OSB}]$ thresholds were significantly higher than the baseline $[\mathrm{COM} / \oslash]$ thresholds for all spacings $\left(F_{1,4}\right.$ $=11.6-133.4 ; p<0.03)$.

For the asynchrony conditions with the CFBs presented continuously $[\mathrm{COM} / \mathrm{CFB}]$, there was a significant overall effect of condition $\left(F_{4,16}=11.47 ; p<0.001\right)$ using the $[\mathrm{OSB} / \oslash]$ reference. Posthoc simple contrasts indicated that thresholds for the narrowest and widest frequency spacings $(1 / 24$ thand 1-octave) did not differ from that for the OSB alone, but thresholds for the $1 / 6$ th- and $1 / 3$ rd-octave spacings did 
$\left(F_{1,4}=30.16-9.03 ; p<0.05\right)$. Thresholds for the $[\mathrm{COM} /$ $\mathrm{CFB}$ ] condition were significantly lower than the [RAN/ RAN] reference for all frequency spacings. A repeated measures ANOVA comparing thresholds in the baseline [COM $\oslash$ ] condition to the asynchronous $[\mathrm{COM} / \mathrm{CFB}]$ condition indicated a significant effect of masker synchrony $\left(F_{1,4}=8.27\right.$; $p<0.05)$, a significant effect of frequency spacing $\left(F_{1,4}\right.$ 29.26; $p<0.001)$, and a significant interaction between these two factors $\left(F_{3,12}=11.98 ; p=0.001\right)$. Analysis of simple effects showed that significant threshold differences existed only for the two widest spacings $\left(F_{1,4}=7.4-36.1 ; p\right.$ $\leqslant 0.05$ ). That is, thresholds in the $[\mathrm{COM} / \mathrm{CFB}]$ condition were elevated with respect to the baseline $[\mathrm{COM} / \oslash]$ condition only for the 1/3rd- and 1-octave spacings.

In summary, the pattern of results from the asynchrony manipulation indicates that the effect of onset/offset asynchrony between OSB and CFBs depends in part on the mode of the asynchrony. When the OSB is presented continuously, any signal detection advantage associated with the presence of CFBs during the observation intervals is eliminated, and in most cases thresholds are elevated relative to the reference $[\mathrm{OSB} / \oslash]$ condition. When the CFBs are presented continuously, the signal detection advantage is reduced only for the wider spacings.

The other stimulus manipulation designed to disrupt use of across-channel cues was that of embedding the gated comodulated complex into a random temporal fringe [COM/ RAN]. In this condition, each of the five bands making up the temporal fringe had an envelope that was independent of that of the others; the results of this condition are shown in Fig. 3, panel B (filled upward triangles). As a comparison to the random temporal fringe, two other temporal fringe conditions were also examined. In one, the outer four bands (flanking bands) of the fringe were comodulated with respect to each other, but independently of the envelope pattern of the band centered at the signal frequency $([\mathrm{COM} / \mathrm{COD}]$, inverted triangles). The purpose of this condition was to create a temporal fringe where, in the context of auditory grouping mechanisms, the OSB might be perceptually segregated from the accompanying noise bands. In the other fringe condition, all five bands remained comodulated, and this condition amounted to a continuous presentation of comodulated noise bands ([COM/COM], filled circles). The purpose of this condition was to determine the extent to which gated/continuous differences existed for comodulated complexes over the range of frequency spacings tested. It is apparent that signal thresholds in both the random and codeviant fringe conditions were similar to those for the reference $[\mathrm{OSB} / \oslash]$ condition, indicating little or no masking release. For the continuous comodulated conditions, thresholds were further reduced for the three wider spacings, such that the masking release for continuous comodulated noise exceeded that for baseline gated comodulated noise.

To test the significance of these patterns, separate repeated measures ANOVAs were undertaken for each fringe condition. Using the $[\mathrm{OSB} / \oslash]$ reference, results for the random temporal fringe $[\mathrm{COM} / \mathrm{RAN}]$ indicated an overall effect of condition $\left(F_{4,16}=21.53 ; p<0.001\right)$, and post hoc simple contrasts indicated that signal threshold in the random fringe was different from that for the OSB alone for all frequency spacings except the $1 / 3$ rd-octave spacing $\left(F_{1,4}\right.$ $=11.33-43.67, p<0.05)$. Note that signal threshold was elevated relative to the OSB reference for the $1 / 24$ th-octave spacing and lower than this reference for the $1 / 6$ th- and 1-octave spacings. Relative to the [RAN/RAN] reference, the [COM/RAN] thresholds were significantly lower for all but the narrowest frequency spacing. Results for the codeviant temporal fringe $[\mathrm{COM} / \mathrm{COD}]$ relative to the $[\mathrm{OSB} / \oslash]$ reference indicated a significant overall effect of condition $\left(F_{4,16}=6.03 ; p<0.005\right)$, and post hoc simple contrasts indicated that only the signal threshold for the narrowest spacing $(1 / 24$ th-octave $)$ was different from reference $\left(F_{1,4}=9.61 ; p\right.$ $<0.05)$. Again, this difference reflected an elevation in signal threshold above reference. The [COM/COD] thresholds did not differ significantly from the [RAN/RAN] reference thresholds for any frequency spacing. Results for the comodulated temporal fringe $[\mathrm{COM} / \mathrm{COM}]$ relative to the $[\mathrm{OSB} / \oslash]$ reference indicated a significant overall effect of condition $\left(F_{4,16}=91.09 ; p<0.001\right)$, and post hoc simple contrasts indicated that signal threshold was lower than that for the OSB reference for all frequency spacings $\left(F_{1,4}\right.$ $=34.72-199.93, p<0.005)$. The $[\mathrm{COM} / \mathrm{COM}]$ thresholds were also significantly lower than the [RAN/RAN] reference thresholds for all frequency spacings. An ANOVA comparing thresholds in the gated comodulated maskers $[\mathrm{COM} / \oslash]$ with those in the continuous gated maskers $[\mathrm{COM} / \mathrm{COM}]$ indicated that thresholds were lower in the continuous mode of presentation for the $1 / 6$ th- and $1 / 3$ rd-octave spacing $\left(F_{1,4}\right.$ $=12.37$ and 32.81, respectively; $p<0.03$ ); the additional masking release failed to reach significance for the $1 / 24$ th-octave spacing $(p=0.28)$ and the 1 -octave spacing $(p=0.07)$.

In summary, this pattern of results indicates that a temporal fringe that does not consist of the same comodulated noise bands as the gated intervals generally eliminates any masking release associated with the gated comodulated bands. When the temporal fringe consists of the same comodulated bands as the gated intervals (i.e., continuous comodulation), the masking release tends to increase for all but the narrowest spectral spacing.

The purpose of this experiment was to determine whether the factors of temporal asynchrony and the presence of a noncomodulated temporal fringe differentially affect multiband masking release depending on whether the frequency spacing of the multiple bands provides predominantly within-channel or across-channel cues. Relative to the $[\mathrm{OSB} / \oslash]$ reference, significant masking release was found with synchronously gated comodulated maskers for the 1/6th-, 1/3rd-, and 1-octave frequency spacings. A temporal asynchrony wherein the CFBs were presented continuously and the OSB was gated on largely eliminated the masking release for the wider spacings but had minimal effect for the $1 / 6$ th-octave spacing. This result is consistent with Dau et al. (2005). For the other mode of temporal asynchrony, wherein the OSB was presented continuously and the flanking bands were gated on, an elevation in threshold above that for the OSB reference occurred for all frequency spacingseven the $1 / 24$ th-octave spacing where no baseline masking 


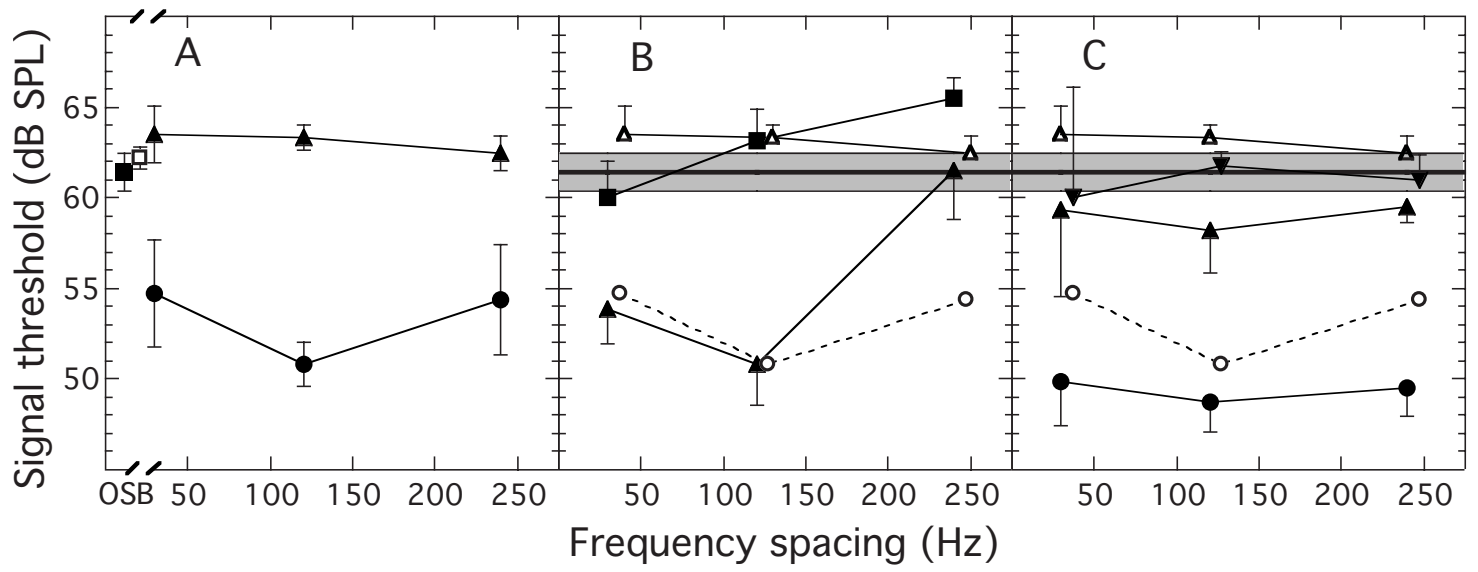

FIG. 4. Mean results from experiment 2. Panel A shows results from the baseline gated comodulated conditions. OSB alone thresholds are shown as filled squares for gated presentation (i.e., $[\mathrm{OSB} / \oslash]$ ) and unfilled squares for continuous presentation (i.e., [OSB/OSB]); thresholds in continuous random maskers [RAN/RAN] as a function of band spacing are shown by triangles. Thresholds in gated comodulated maskers [COM/ $\oslash$ are shown as circles. Panel B shows results from the asynchrony conditions, and panel $\mathrm{C}$ shows results from the temporal fringe conditions. Panel $\mathrm{B}$ : filled squares=[COM/OSB]; filled triangles $=[\mathrm{COM} / \mathrm{CFB}]$. Panel $\mathrm{C}$ : filled triangles $=[\mathrm{COM} / \mathrm{RAN}]$; filled inverted triangles $=[\mathrm{COM} / \mathrm{COD}]$; filled circles $=[\mathrm{COM} / \mathrm{COM}]$. In each panel, replotted from panel A, are the $[\mathrm{OSB} / \oslash]$ reference (the thick horizontal line bounded by a \pm 1 standard deviation shaded region), the [RAN/RAN] reference (open triangles), and the $[\mathrm{COM} / \mathrm{OSB}]$ baseline (open circles). Error bars are 1 standard deviation.

release was observed. The baseline masking releaseincluding that for the 1/6th-octave spacing-was also largely, or completely, eliminated by the presence of a random or codeviant temporal fringe. A comodulated fringe generally served to increase the magnitude of masking release when it was present in the baseline conditions. For the [RAN/RAN] reference, where a masking release is now observed for the narrowest $1 / 24$ th-octave spacing, a broadly similar picture emerges of the effects of temporal asynchrony/fringe as a function of frequency spacing.

Before considering the implications of these findings for the hypothesis being tested in detail, it is prudent to note a limitation in the stimulus configurations used in experiment 1. As in Dau et al. (2005), the frequency spacings were defined on a $\log$ scale. Whereas this allowed an adjustment in frequency proximity that was approximately uniform in cochlear space, it did not result in configurations that accentuated the cues thought to be important for within-channel processing. As noted in the Introduction, a primary cue for the presence of a signal in a complex of closely spaced comodulated bands is thought to be its disruption of the inherent beating pattern between bands (Schooneveldt and Moore, 1987). If so, then the use of logarithmic spacing is not favorable to this cue since the beat frequencies are not uniform across bands. Instead, linear spacings would be more conducive to the generation of a signal-induced, beat-disruption cue since the pattern of component interactions between neighboring bands are uniform across bands. A companion experiment was therefore undertaken that employed linear spacings of comodulated bands. Its purpose was to improve the cues thought to result in masking release for proximal frequency spacings in order to further clarify the effects of temporal asynchrony and random temporal fringe on withinchannel processing of comodulated bands. It was anticipated that a comparison of the results of the two experiments would give a more complete picture of within- and acrosschannel processing in CMR, and address more fully the hy- pothesis that the factors of temporal asynchrony and random temporal fringe undermine masking release associated with across-channel processes but not within-channel processes.

\section{EXPERIMENT 2. MULTIBAND CMR FOR LINEAR SPACING}

\section{A. Method}

The stimulus parameters and procedure were the same as experiment 1 except that the frequency spacings of the five-band maskers were linear rather than logarithmic. In addition, the random reference condition was gated [RAN/ $\oslash$ ] instead of continuous [RAN/RAN]. ${ }^{5}$ The frequency spacings were 30,120 , and $240 \mathrm{~Hz}$, resulting in center frequencies, respectively, of 940, 970, 1000, 1030, and $1060 \mathrm{~Hz} ; 760$, 880, 1000, 1120, and $1240 \mathrm{~Hz}$; and 520, 760, 1000, 1240, and $1480 \mathrm{~Hz}$. These separations were selected to be globally similar to the $1 / 24$ th-, $1 / 6$ th- and $1 / 3$ rd-octave spacings of experiment 1 . The same observers participated as in experiment 1 .

\section{B. Results and discussion}

The results of experiment 2 are shown in Fig. 4, following the same labeling conventions as Figs. 2 and 3. In panel $\mathrm{A}$, it can be seen that signal thresholds did not differ between the gated $[\mathrm{OSB} / \oslash]$ and continuous $[\mathrm{OSB} / \mathrm{OSB}]$ references $\left(t_{4}=1.49 ; p=0.19\right)$. It can also be seen that thresholds in the baseline gated comodulated conditions $[\mathrm{COM} / \oslash]$ were reduced relative to either the OSB alone reference or the random band reference [RAN/ $\oslash$; i.e., a masking release occurred for all frequency spacings. This was confirmed with repeated measures ANOVAs which indicated that thresholds in the comodulated maskers were each lower than either the $[\mathrm{OSB} / \oslash]$ reference $\left(F_{1,4}=28.63-147.87 ; p<0.01\right)$ or the $[\mathrm{RAN} / \oslash]$ reference $\left(F_{1,4}=101.1 ; p<0.001\right)$.

The effect of onset asynchrony is shown in panel B of Fig. 4. When the OSB was presented continuously and the 
flanking bands were gated on during the listening intervals ([COM/OSB], filled squares), thresholds were comparable to, or higher than, the $[\mathrm{OSB} / \oslash]$ reference threshold. When the flanking bands were presented continuously and the OSB was gated ([COM/CFB], filled triangles), thresholds were not changed for the two narrowest spacings ( 30 and $120 \mathrm{~Hz}$ ), but rose to the reference levels for the widest spacing $(240 \mathrm{~Hz})$. This pattern was confirmed with repeated measures ANOVAs. For the [COM/OSB] asynchrony relative to the $[\mathrm{OSB} / \oslash]$ reference, there was a significant effect of condition $\left(F_{3,12}=13.21 ; p<0.001\right)$. Post hoc contrasts indicated that thresholds did not differ from the reference threshold for the 30 and $120 \mathrm{~Hz}$ spacings $\left(F_{1,4}=2.22\right.$ and $\left.2.41 ; p \geqslant 0.20\right)$ but did differ significantly for the $240 \mathrm{~Hz}$ spacing $\left(F_{1,4}\right.$ $=200.05 ; p<0.001)$. Relative to the $[$ RAN/ $\oslash]$ reference, there was a significant interaction between the [COM/OSB] and $[\mathrm{RAN} / \oslash]$ conditions as a function of frequency spacing $\left(F_{2,8}=11.27 ; p<0.005\right)$; analysis of simple effects indicated that thresholds for the asynchronous masker were lower for the $30 \mathrm{~Hz}$ spacing and higher for the $240 \mathrm{~Hz}$ spacing $\left(F_{1,4}\right.$ $=14.6$ and 23.6, respectively; $p<0.02$ ), but did not differ for the $120 \mathrm{~Hz}$ spacing. For the [COM/CFB] asynchrony referenced to $[\mathrm{OSB} / \oslash]$ thresholds, there was a significant effect of condition $\left(F_{3,12}=30.53 ; p<0.001\right)$. Post hoc contrasts showed that thresholds were significantly lower than the reference threshold for the two narrowest spacings $\left(F_{1,4}\right.$ $=66.41$ and $58.28 ; p<0.01)$ but not for the $240 \mathrm{~Hz}$ spacing $\left(F_{1,4}=0.01 ; p=0.94\right)$. Relative to the $[\mathrm{RAN} / \oslash]$ reference, there was a significant interaction between the $[\mathrm{COM} / \mathrm{CFB}]$ and $[\mathrm{RAN} / \oslash]$ conditions as a function of frequency spacing $\left(F_{2,8}=21.71 ; p<0.001\right)$; analysis of simple effects indicated that thresholds were lower for the $30 \mathrm{~Hz}$ spacing and the $120 \mathrm{~Hz}$ spacing $\left(F_{1,4}=75.81\right.$ and 101.77 , respectively; $p$ $<0.001$ ), but not for the $240 \mathrm{~Hz}$ spacing.

The effect of temporal fringe is shown in panel C of Fig. 4. For the random temporal fringe ([COM/RAN], upward triangles), thresholds approach the reference thresholds, although some masking release appears to remain. For the codeviant fringe ([COM/COD], inverted triangles), thresholds largely coincide with the reference thresholds. For the comodulated fringe ([COM/COM], filled circles), thresholds are further reduced relative to baseline. The relatively large standard deviations associated with the data for $30 \mathrm{~Hz}$ spacing of the random and codeviant fringe conditions should be kept in mind when considering the data analysis. Individual differences were striking for these conditions and, to highlight this, the individual results are shown in Fig. 5. It can be seen that, for three of the five observers, the random and deviant temporal fringe manipulations resulted in elevated thresholds relative to the baseline gated comodulated condition. However, for the remaining two listeners, the fringe manipulations had little effect. This degree of individual variability in the effect of the temporal fringe for the narrowest $30 \mathrm{~Hz}$ spacing suggests that the threshold elevationwhen it is observed-is not due to factors associated with energetic masking.

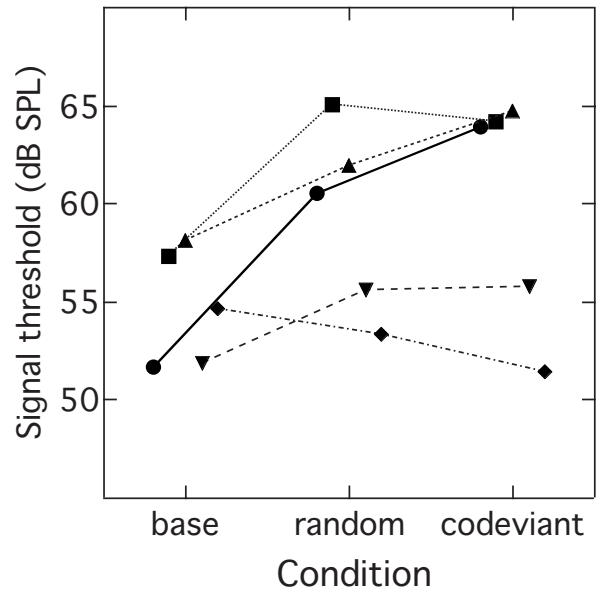

FIG. 5. Individual data for the $30 \mathrm{~Hz}$ spacing of experiment 2. Thresholds are shown for the baseline masking release condition, the random temporal fringe, and the codeviant temporal fringe.

\section{GENERAL DISCUSSION}

The stimulus conditions of experiments 1 and 2 were designed to provide thresholds under comparable conditions for logarithmic and linear masker spacing. The motivation for experiment 2 was to augment the within-channel beating cue by using linear, rather than logarithmic, spacing. The $30 \mathrm{~Hz}$ linear spacing resulted in center frequencies that were similar to those for the $1 / 24$ th-octave spacing of experiment 1 ; whereas no masking release was observed for the $\log$ spacing, a $6.7 \mathrm{~dB}$ masking release was observed for the linear spacing. This suggests that linear spacing provides a stronger within-channel beating cue than logarithmic spacing does. This conclusion is supported by thresholds obtained at the next wider spacing in both experiments, which were also thought to provide robust within-channel cues. Whereas the $1 / 6$ th-octave spacing of experiment 1 led to about a $6.5 \mathrm{~dB}$ masking release, the $120 \mathrm{~Hz}$ linear spacing of experiment 2 resulted in a masking release of about $10.5 \mathrm{~dB}$. In contrast, the $1 / 3$ rd-octave spacing of experiment 1 and the $240 \mathrm{~Hz}$ spacing of experiment 2 both gave similar magnitudes of masking release $(6.7$ and $7.0 \mathrm{~dB}$, respectively). These two spacings had similar spectral distributions, and both were expected to provide primarily across-channel cues. The similar masking releases for these linear and logarithmic spacings suggest that the detection cue here was not associated with a disruption of beat patterns due to the addition of a signal tone.

A final piece of evidence which supports the use of a beating cue in configurations where the frequency spacing is both narrow and linear comes from a supplementary condition that used a modified OSB. Recall that the CFBs were constructed by assigning to their real and imaginary components the same values as assigned to the corresponding components in the OSB. As a result of this, the beating patterns between the components of any neighboring linearly spaced bands were identical. In the supplemental condition, the OSB was modified such that its spectral profile was changed yet its envelope remained comodulated with that of the flanking bands. To achieve this, the frequency spectrum of the OSB was modified by multiplying the phase values by -1 and 
reversing the assignment of both magnitude and phase values as a function of component number (see Richards, 1988). Despite the maintenance of comodulation, the beating patterns between the OSB components and those of neighboring comodulated bands were now different from those between the components of the flanking bands themselves. The aim of this manipulation was to undermine the strength of the cue wherein the signal disrupts the uniform beating pattern of the comodulated masker complex. The modified masker was presented in continuous mode, and therefore the appropriate comparison is to the original comodulated complex also presented continuously (i.e., [COM/COM]). The average signal threshold masked by the original comodulated complex was $49.9 \mathrm{~dB}$ SPL, whereas the average threshold for the comodulated complex with the "mirror image" OSB was $58.4 \mathrm{~dB}$ SPL. This $8.5 \mathrm{~dB}$ threshold elevation when the beating cue was diminished provides further support for the contention that the linear spacings of experiment 2 facilitated the use of a beating disruption cue for signal detection under conditions of proximal spacing. In summary, the aim of accentuating the availability of temporal beating cues by using linear spacing in experiment 2 appears to have been successful.

The greater salience of within-channel processing cues in experiment 2 contributes to the interpretation of the comparative data patterns across the two experiments. For the four spectral spacings of experiments 1 and 2 where predominantly within-channel processing was expected $(1 / 24$ th-octave, $1 / 6$ th-octave, $30 \mathrm{~Hz}, 120 \mathrm{~Hz})$, a temporal asynchrony wherein the flanking bands were presented continuously and the OSB was gated had minimal effect on signal threshold. In contrast, for the spacings conducive to across-channel processing in the two experiments (1/3rd-octave, 1-octave, $240 \mathrm{~Hz})$, this type of temporal asynchrony largely eliminated any masking release. Taken together, this pattern of results is consistent with the contention that within-channel processing is not sensitive to asynchrony among comodulated noise bands, whereas across-channel processing is. This, in turn, supports the hypothesis that across-channel contributions to auditory grouping can be disrupted by onset asynchrony.

However, the association between the onset asynchrony effect and a reliance on across-channel cues is not supported by results obtained with the alternative type of onset asynchrony, wherein the OSB was presented continuously and the flanking bands were gated. Here, irrespective of frequency spacing, thresholds were elevated to levels as high as, or higher than, the reference threshold. The elevation of threshold above the OSB reference threshold has been observed in previous work testing both adults (Grose and Hall, 1993) and children (Hall et al., 1997) but is not uniformly found (Grose and Hall, 1996). It should be noted that in all these previous studies the signal onset was delayed relative to the masker onset by 100-200 ms; here, the signal onset was synchronous with the gated masker onset. As such, the increased masking bears some resemblance to the "transient masking" associated with the onset of a remote masker as measured by Bacon and Moore (1987), although that study pertained mainly to brief signals/maskers. One ramification of this resemblance to transient masking is that it suggests that the threshold elevation associated with the gated flanking bands might not be restricted to CFBs; that is, similar threshold elevations might be observed with gated random flanking bands. However, this condition [RAN/OSB] was not tested in this study. The observation that the elevation of threshold above the reference threshold tended to be largest for the $1 / 3$ rd-octave (logarithmic) and $240 \mathrm{~Hz}$ (linear) spacings might suggest that some form of across-channel masking contributes to the effect.

The temporal fringe manipulation had a generally uniform effect across the two experiments. When the fringe consisted of a set of random noise bands, or a set of CFBs plus an OSB with an independent fluctuation pattern (codeviant), masking release was largely eliminated irrespective of the frequency spacing of the noise bands. This general result runs counter to the suggestion that the disruptive effects of a noncomodulated temporal fringe are specific to acrosschannel cues and should therefore not affect signal threshold in cases where performance is based on within-channel cues. Note again that for the linear spacing of bands there were large individual differences at the narrowest spacing of the noncomodulated fringe, with two of the five observers being minimally affected by the fringe.

In order to gain insight into the overall pattern of results, it is useful to focus separately on possible within-channel and across-channel contributions to that pattern. Beginning first with within-channel contributions, recall that one hypothesis was that masking release related to within-channel cues would not be reduced by either the random temporal fringe or by onset asynchrony. The results of the narrow frequency spacing conditions were not entirely consistent with this hypothesis. Whereas the hypothesis was supported for the asynchrony condition where the flanking bands were continuous, it was not supported for the asynchrony condition where the OSB was continuous, nor for the condition where there was a random temporal fringe. We will briefly consider a possible account for the pattern of results obtained for the narrow spacing conditions, where performance is probably related to the processing of a within-channel cue. In this account, it is assumed that the listener monitors the regular beating pattern arising from the interaction among comodulated bands and bases detection on the change in beating pattern that occurs when the signal is added. In the asynchrony case where the CFBs are present continuously, the listener can monitor the pattern of the interactions among the CFBs, a pattern that is regular during both the no-signal intervals and the interstimulus intervals. This is consistent with the finding that performance was relatively good in the condition where the flanking bands were presented continuously and the OSB was gated on. Use of a beating cue is also consistent with the relatively poor performance in the asynchrony condition where the OSB was presented continuously, in that the stimulus surrounding the listening intervals contained no regular beating pattern due to the fact that a single noise band was present. The different effects of onset asynchrony as a function of masker spacing observed here could depend on the number of flanking bands. For example, if there were only two noise bands, one centered on the signal and one flanking band, then the different effects of gating 
mode would not be expected to occur because beating between comodulated bands would not occur either for a continuous flanking band or a continuous OSB.

Use of a cue based on envelope beats is also consistent with the finding that performance with narrow frequency spacing was poor for the case where the fringe consisted of either random bands or CFBs plus an OSB with an independent fluctuation pattern (codeviant). In the case of the random fringe, the stimulus surrounding the listening intervals contained no consistent beating pattern due to the fact that the flanking bands had random envelopes. In the codeviant fringe condition, the OSB would diminish the regularity of the beating cue even in the absence of a pure tone signal. In both cases, stimulus features outside the listening interval fail to form a stream of consistent envelope beats.

Note that in the above accounts, it is assumed that the listener cannot simply base detection on the information within the observation intervals (where all bands are comodulated), due to some sort of "carry over" effect wherein the pattern of energy surrounding the listening intervals prevents the listener from optimally processing the beating pattern within the listening interval. Moreover, a listener's susceptibility to this carry over varies markedly as demonstrated by the extensive individual variability seen for the narrow $30 \mathrm{~Hz}$ linear spacing (see Fig. 5). One of the observers who was unaffected by the random and codeviant fringes in this configuration reported that the detection cue for the signal was a disruption in the regularity of the beat percept during the comodulated core interval, and that this cue remained salient even in the presence of a noncomodulated temporal fringe.

Turning now to across-channel contributions to the overall pattern of results, recall that part of the motivation for this study was based on the idea that auditory grouping is a precondition to across-channel CMR, and that the effects of onset asynchrony would be relatively great for masking release based on across-channel analysis. In contrast, the presence of random bands surrounding the observation intervals was thought to disrupt the listener's ability to make use of coherent modulation during the observation intervals independent of grouping. For the wider masker spacings, all manipulations tested elevated thresholds (except for the continuous comodulated condition). This effect could be due to reduced grouping strength in the case of onset asynchrony and some other factors in the temporal fringe conditions. However, these effects could also be attributed to some other common factor. One possibility is the regularity of spectrotemporal coherence. Here, the cue in across-channel CMR is characterized as a discontinuity in the dynamic spectrum of the stimulus. Addition of a pure tone signal can result in a spectral peak at the signal frequency when energy at other frequencies is at a modulation minimum, a cue that could be the basis of a detection process sometimes referred to as listening in the dips. It is possible that such an acrosschannel cue can be disrupted by spectrotemporal irregularities that occur outside the observation intervals. For example, random bands surrounding the observation intervals expose the listener to a variable spectrum, where peaks in the spectrum are associated with different frequency regions as a function of time. These peaks could impede the formation, or stability, of a no-signal template (in this case a flat spectrum that coherently fluctuates in level as a function of time). Similarly, asynchronous gating introduces radical changes in the spectrum of the masker that could interfere with cues based on more subtle spectral cues associated with addition of a pure tone signal.

The importance of spectrotemporal coherence for the use of an across-channel cue and the importance of envelope beat regularity as a function of time for the use of withinchannel cues could be described in similar terms. Whereas spectrotemporal coherence may facilitate detection of a change in the spectrum, consistency of envelope statistics in a single auditory channel output could facilitate detection of a change in that temporal pattern. Features of a random stimulus surrounding the observation intervals, such as reduced modulation depth (within-channel) or spectral variability (across-channel), could resemble the stimulus features associated with addition of a pure tone signal. Asynchronous gating across bands could likewise interfere with formation of a no-signal baseline against which to compare the observation interval. It is therefore possible that performance based on either within- or across-channel cues is disrupted by manipulation of the spectrotemporal coherence of the stimulus surrounding the observation intervals for rather similar reasons.

The implication of these results for previous studies deserves comment. Dau et al. (2005) manipulated temporal asynchrony to differentiate within- and across-channel contributions to CMR. They hypothesized that the use of acrosschannel cues contributing to masking release for comodulated noise would be affected by across-frequency grouping mechanisms, but that within-channel mechanisms would not. In their experiment, they used onset asynchrony wherein the flanking bands were gated on before, and gated off after, the OSBs. The CMR declined under conditions of temporal asynchrony only for stimulus complexes made up of widely spaced noise bands, a result that supported the hypothesis. The results of the present investigation replicate this result, but indicate that support for the hypothesis should be tempered in light of the results obtained with the other mode of onset asynchrony. Using similar reasoning as Dau et al. (2005), Grose et al. (2005b) incorporated the manipulation of a random temporal fringe as a test for across-channel processing in CMR. They proposed that the presence of a random temporal fringe should reduce the likelihood of using available across-channel cues and should therefore diminish across-channel CMR. The results of the present investigation caution that sensitivity of signal threshold to this manipulation is not a specific indicator of across-channel processing, since a disruption to masking release due to within-channel processing also results from the presence of a noncomodulated temporal fringe.

\section{SUMMARY AND CONCLUSIONS}

The purpose of this investigation was to determine whether within- and across-channel contributions to masking release in the multiband CMR paradigm could be differentiated via manipulations of the stimulus characteristics in the 
interstimulus interval. Two stimulus manipulations were examined: (1) onset asynchrony between the OSB and the $\mathrm{CFBs}$, and (2) the presence of a random temporal fringe surrounding the core comodulated bands. The availability of within- and across-channel cues was manipulated by varying the frequency spacing of the narrow bands of noise making up the comodulated complex. Both logarithmic and linear spacings were employed. It was hypothesized that the two stimulus manipulations would disrupt masking release due to across-channel cues but not masking release due to withinchannel cues.

The overall pattern of results did not wholly support the hypothesis. When the OSB was gated on during the observation intervals and the CFBs were presented continuously the results were compatible with the hypothesis: masking release was not affected for stimuli providing robust within-channel cues, but it was disrupted for stimuli providing robust acrosschannel cues. However, presenting the OSB continuously and gating the flanking bands elevated thresholds, irrespective of frequency spacing. The second stimulus manipulation (random temporal fringe) also generated results that did not support the hypothesis: masking release was largely eliminated by the presence of a random temporal fringe irrespective of the frequency spacing of the noise bands making up the masking complex.

In conclusion, this investigation has demonstrated that a detection advantage for a tone masked by a complex of comodulated narrow bands of noise can be observed under conditions of predominantly within- or cross-channel processing. Identifying the mechanisms underlying the masking release remains a challenge. Manipulation of stimulus features in the interstimulus interval that were previously thought to disrupt only across-channel processes has been shown to also disrupt within-channel processes under some conditions.

\section{ACKNOWLEDGMENTS}

This work was supported by NIH NIDCD R01DC01507 and R01-DC007391. Helpful comments on previous drafts were provided by Brian Moore and two anonymous reviewers.

${ }^{1}$ For the band-widening method of measuring CMR, Verhey et al. (1999) showed that within-channel cues can provide the predominant cues for signal detection.

${ }^{2}$ MDI refers to the phenomenon wherein detection of modulation carried by one tone is disrupted by the presence of a spectrally remote tone that is modulated at a suprathreshold level (Yost and Sheft, 1989).

${ }^{3} \mathrm{CDD}$ refers to the phenomenon wherein detection threshold for a narrow band of noise is typically higher in the presence of a complex of comodulated narrow bands of noise than a complex of noise bands that do not share the same fluctuation pattern as the target band (Cohen and Schubert, 1987; McFadden, 1987).

${ }^{4}$ Although Dau et al. (2005) did not test conditions where the OSB was gated on before, and gated off after, the CFBs, they extracted data for this condition from the Grose and Hall (1993) study. Thus, their identification of a discrepancy between their findings and those of Grose and Hall (1993) is due to a comparison of dissimilar conditions. Like Dau et al.,
(2005) Grose and Hall (1993) did not observe a total elimination of masking release when flanker onset preceded on-signal masker onset.

${ }^{5}$ Pilot data indicated that there was no difference in masked threshold between gated and continuous random masking bands.

Bacon, S. P., and Moore, B. C. (1987). "Transient masking and the temporal course of simultaneous tone-on-tone masking," J. Acoust. Soc. Am. 81, 1073-1077.

Berg, B. G. (1996). "On the relation between comodulation masking release and temporal modulation transfer functions," J. Acoust. Soc. Am. 100, 1013-1023.

Cohen, M. F., and Schubert, E. D. (1987). "The effect of cross-spectrum correlation on the detectability of a noise band," J. Acoust. Soc. Am. 81, 721-723.

Dau, T., Ewert, S. D., and Oxenham, A. J. (2005). "Effects of concurrent and sequential streaming in comodulation masking release," in Auditory Signal Processing: Physiology, Psychoacoustics, and Models, edited by D. Pressnitzer, A. de Cheveigne, S. McAdams, and L. Collet (Springer, New York).

Grose, J. H., and Hall, J. W., III. (1996). "Cochlear hearing loss and the processing of modulation: Effects of temporal asynchrony," J. Acoust. Soc. Am. 100, 519-527.

Grose, J. H., and Hall, J. W., III. (1993). "Comodulation masking release: Is comodulation sufficient?," J. Acoust. Soc. Am. 93, 2896-2902.

Grose, J. H., Hall, J. W., III, and Buss, E. (2005a). "Across-channel spectral processing," Int. Rev. Neurobiol. 70, 87-119.

Grose, J. H., Hall, J. W., III, Buss, E., and Hatch, D. R. (2005b). "Detection of spectrally complex signals in comodulated maskers: Effect of temporal fringe," J. Acoust. Soc. Am. 118, 3774-3782.

Hall, J. W., III, Buss, E., and Grose, J. H. (2006). "Comodulation detection differences for fixed-frequency and roved-frequency maskers," J. Acoust. Soc. Am. 119, 1021-1028.

Hall, J. W., and Grose, J. H. (1991). "Some effects of auditory grouping factors on modulation detection interference (MDI)," J. Acoust. Soc. Am. 90, 3028-3036.

Hall, J. W., Grose, J. H., and Dev, M. B. (1997). "Auditory development in complex tasks of comodulation masking release," J. Speech Lang. Hear. Res. 40, 946-954.

Hall, J. W., Haggard, M. P., and Fernandes, M. A. (1984). "Detection in noise by spectrotemporal pattern analysis," J. Acoust. Soc. Am. 76, 50-56. Hatch, D. R., Arne, B. C., and Hall, J. W. (1995). "Comodulation masking release (CMR): Effects of gating as a function of number of flanking bands and masker bandwidth," J. Acoust. Soc. Am. 97, 3768-3774.

McFadden, D. (1987). "Comodulation detection differences using noiseband signals," J. Acoust. Soc. Am. 81, 1519-1527.

Mendoza, L., Hall, J. W., III, and Grose, J. H. (1998). "Comodulation masking release: The effect of the characteristics of noisebands presented before and after a signal," J. Acoust. Soc. Am. 103, 2843.

Moore, B. C., and Borrill, S. J. (2002). "Tests of a within-channel account of comodulation detection differences," J. Acoust. Soc. Am. 112, 2099-2109.

Moore, B. C., and Shailer, M. J. (1992). "Modulation discrimination interference and auditory grouping," Philos. Trans. R. Soc. London, Ser. B 336, 339-346.

Oxenham, A. J., and Dau, T. (2001). "Modulation detection interference: Effects of concurrent and sequential streaming," J. Acoust. Soc. Am. 110, 402-408.

Richards, V. M. (1988). "Components of monaural envelope correlation perception," Hear. Res. 35, 47-58.

Schooneveldt, G. P., and Moore, B. C. J. (1987). "Comodulation masking release (CMR): Effects of signal frequency, flanking-band frequency, masker bandwidth, flanking-band level, and monotic versus dichotic presentation flanking band," J. Acoust. Soc. Am. 82, 1944-1956.

Verhey, J. L., Dau, T., and Kollmeier, B. (1999). "Within-channel cues in comodulation masking release (CMR): Experiments and model predictions using a modulation-filterbank model," J. Acoust. Soc. Am. 106, 2733-2745.

Verhey, J. L., Pressnitzer, D., and Winter, I. M. (2003). "The psychophysics and physiology of comodulation masking release," Exp. Brain Res. 153, 405-417.

Yost, W. A., and Sheft, S. (1989). "Across-critical-band processing of amplitude-modulated tones," J. Acoust. Soc. Am. 85, 848-857. 